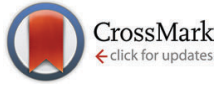

Cite this: Phys. Chem. Chem. Phys., 2014, 16, 26279

Received 5th June 2014 Accepted 17th October 2014

DOI: $10.1039 / c 4 c p 02480 j$

www.rsc.org/pccp

\title{
Convective dynamics of traveling autocatalytic fronts in a modulated gravity field
}

\author{
Dezső Horváth, ${ }^{\star a b}$ Marcello A. Budroni, ${ }^{\star c d}$ Péter Bába, ${ }^{a}$ Laurence Rongy, ${ }^{c}$ \\ Anne De Wit, ${ }^{c}$ Kerstin Eckert, ${ }^{e}$ Marcus J. B. Hauser ${ }^{f}$ and Ágota Tóth ${ }^{a}$
}

\begin{abstract}
When traveling in thin solution layers, autocatalytic chemical fronts may be deformed and accelerated by convective currents that develop because of density and surface tension gradients related to concentration and thermal gradients across the front. On earth, both buoyancy and Marangoni related flows can act in solution layers open to the air while only buoyancy effects operate in covered liquid layers. The respective effects of density and surface tension induced convective motions are analysed here by studying experimentally the propagation of autocatalytic fronts in uncovered and covered liquid layers during parabolic flights in which the gravity field is modulated periodically. We find that the velocity and deformation of the front are increased during hyper-gravity phases and reduced in the micro-gravity phase. The experimental results compare well with numerical simulations of the evolution of the concentration of the autocatalytic product coupled to the flow field dynamics described by Navier-Stokes equations.
\end{abstract}

\section{Introduction}

Autocatalytic fronts resulting from the interplay between diffusion and an autocatalytic reaction are well-known examples of out-ofequilibrium self-organization. They usually propagate with a constant shape and at a constant speed in gels. ${ }^{1-3}$ However, if the reaction takes place in a solution, the gradients of concentrations and temperature across the front can result in a modification of the physical parameters of the fluid, such as density or surface tension. These changes can trigger natural convection, which has long been experimentally evidenced to, in turn, deform autocatalytic fronts and accelerate their propagation. ${ }^{4-20}$

In vertically-oriented closed systems where the reactor is entirely filled with the reacting solution and the front travels parallel to the gravity field, convective flows can be induced by density differences only. The resulting density fingering of the front has been well characterized both experimentally and

\footnotetext{
${ }^{a}$ Department of Physical Chemistry and Materials Science, University of Szeged, Rerrich Béla tér 1., Szeged, H-6720, Hungary.E-mail: horvathd@chem.u-szeged.hu; Fax: +36-62-546-482; Tel: +36-62-544-614

${ }^{b}$ Department of Applied and Environmental Chemistry, University of Szeged, Rerrich Béla tér 1., Szeged, H-6720, Hungary

${ }^{c}$ Nonlinear Physical Chemistry Unit, CP 231, Faculté des Sciences, Université libre de Bruxelles (ULB), 1050 Brussels, Belgium

${ }^{d}$ Department of Chemistry and Pharmacy, University of Sassari, 07100, Sassari, Italy

${ }^{e}$ Institute of Fluid Mechanics, Technische Universität Dresden, D-01062 Dresden, Germany

${ }^{f}$ Biophysics Group, Otto-von-Guericke-Universität Magdeburg, D-39106 Magdeburg, Germany
}

theoretically. ${ }^{21-29}$ If the front propagates in a horizontal solution layer open to the air, the situation is, however, more complex since both Marangoni (surface tension-driven) and buoyancy (densitydriven) effects can come into play. To try to clarify this situation, numerous theoretical works have been devoted to the study of chemically-driven flows around horizontally propagating fronts and to the effect the flow has on the shape and speed of these fronts. These theoretical studies can be classified depending on the type of flow considered: buoyancy-driven, ${ }^{12,30-39}$ Marangoni-driven, ${ }^{40-47}$ or a combination of both flows. ${ }^{12,19,48,49}$

In the case of pure buoyancy-driven flows, a good agreement exists between numerical simulations and experiments in closed reactors, both for isothermal ${ }^{31,35,38,39}$ and exothermic fronts. ${ }^{37,38,50}$ On the other hand, such a comparison is not available yet in the case of pure surface tension-driven flows because of the difficulty to isolate Marangoni effects from gravity effects on earth. In this context, studies under micro-gravity conditions are crucial to understand the role of both types of convective flows in the front dynamics. They have already proven very helpful in explaining the dynamics of $\mathrm{A}+\mathrm{B} \rightarrow \mathrm{C}$ reactions in immiscible two-layer systems. ${ }^{51,52}$ A combination of parabolic flight experiments and numerical simulations of modulated gravitational acceleration has recently allowed to highlight the role of buoyancy-driven vortices in the behavior of $\mathrm{A}+\mathrm{B} \rightarrow \mathrm{C}$ fronts. ${ }^{53}$ In the case of chemical waves, the effect of density gradients across fronts of the Belousov-Zhabotinsky reaction were shown to die out under micro-gravity conditions in drop towers. ${ }^{54,55}$

In the present paper, we study the dynamics of autocatalytic fronts of the iodate-arsenous acid (IAA) reaction in horizontal 
solution layers with a combination of experiments in parabolic flights and numerical simulations. The IAA reaction possesses rich dynamic behaviors and can give rise to traveling conversion fronts when coupled to diffusion in spatially extended systems. ${ }^{1,2}$ This autocatalytic reaction can be described quantitatively by a one-variable cubic kinetics in some concentration ranges of the reactants. The reaction can be treated as isothermal as Martin et $a .^{50}$ could not detect any heat pulse around traveling IAA fronts using a quantitative IR measurement technique with a $10 \mathrm{mK}$ resolution. They have further computed density variations due to either solutal or thermal effects and concluded that the latter ones are negligible so that the dynamics of IAA fronts are unaffected by temperature effects.

In a recent paper, we have theoretically investigated the combined effect of solutal Marangoni and buoyancy-driven flows on the horizontal propagation of such an isothermal front. ${ }^{49}$ Aiming at a general classification, we have considered both the cases when surface and bulk forces act cooperatively to deform a chemical front and when they have an antagonistic contribution to the flow direction. In the cooperative case, asymptotic regimes are found where the deformed front propagates at a constant speed and with a steady shape, whereas in the antagonistic case, both steady and oscillatory regimes can be observed. Here, our goal is to provide a detailed comparison between experiments and simulations in the specific case of IAA fronts (cooperative regime) propagating in modulated gravity. By comparing measurements in closed cells with the ones obtained in open cells, we aim to discriminate between surface tension-driven and density-driven flows.

We first describe the chemical system and introduce the experimental setup and the theoretical Reaction-DiffusionConvection (RDC) model we have used. Next, we present the results of the parabolic flights in both cases of a closed reactor and of a reactor open to air. A comparison between the front behavior observed in both systems confirms the relative importance of buoyancy and Marangoni effects on the dynamics. The numerical simulations are in excellent agreement with the experimental behavior and make clear the underlying changes in the structure of the flow field. We conclude the article by a comparison between the different cases and between the experiments and numerical simulations.

\section{Chemical system and experimental setup}

The iodate-arsenous acid reaction is a model reaction of cubic autocatalysis with iodide and hydrogen ions being the autocatalysts. Two main reactions describe the mechanism. ${ }^{4,5}$ The first step is the Dushman reaction, where iodate and iodide ions react under acidic condition:

$$
\mathrm{IO}_{3}{ }^{-}+5 \mathrm{I}^{-}+6 \mathrm{H}^{+} \rightarrow 3 \mathrm{I}_{2}+3 \mathrm{H}_{2} \mathrm{O} \text {. }
$$

The second step is the Roebuck reaction, in which arsenous acid is oxidized by iodine:

$$
\mathrm{H}_{3} \mathrm{AsO}_{3}+\mathrm{I}_{2}+\mathrm{H}_{2} \mathrm{O} \rightleftharpoons 2 \mathrm{I}^{-}+\mathrm{H}_{2} \mathrm{AsO}_{4}{ }^{-}+3 \mathrm{H}^{+} .
$$

The net reaction depends on the ratio of the initial reactant concentrations $R=\left[\mathrm{H}_{3} \mathrm{AsO}_{3}\right]_{0} /\left[\mathrm{KIO}_{3}\right]_{0}$.

For $R>3$, iodide ion is the product, according to

$$
\mathrm{IO}_{3}{ }^{-}+3 \mathrm{H}_{3} \mathrm{AsO}_{3} \rightarrow \mathrm{I}^{-}+3 \mathrm{H}_{2} \mathrm{AsO}_{4}{ }^{-}+3 \mathrm{H}^{+}
$$

while for $R<5 / 2$, only molecular iodine is produced as

$$
2 \mathrm{IO}_{3}{ }^{-}+5 \mathrm{H}_{3} \mathrm{AsO}_{3} \rightarrow \mathrm{I}_{2}+5 \mathrm{H}_{2} \mathrm{AsO}_{4}{ }^{-}+\mathrm{H}_{2} \mathrm{O}+3 \mathrm{H}^{+} \text {. }
$$

At $R=8 / 3$, the formation of triiodide ion is at maximum considering the $\mathrm{I}_{2}+\mathrm{I}^{-} \rightleftharpoons \mathrm{I}_{3}{ }^{-}$equilibrium, leading to

$$
3 \mathrm{IO}_{3}{ }^{-}+8 \mathrm{H}_{3} \mathrm{AsO}_{3} \rightarrow \mathrm{I}_{3}{ }^{-}+8 \mathrm{H}_{2} \mathrm{AsO}_{4}{ }^{-}+\mathrm{H}_{2} \mathrm{O}+6 \mathrm{H}^{+} \text {. }
$$

In the reaction, iodine is surface active and decreases the surface tension of the solution behind the reaction front. Since iodine is also a volatile species, its evaporation might affect the distortion of the front at the liquid-gas interface. The ratio between the initial concentrations of arsenous acid and potassium iodate $(R=2.8)$ has been selected to avoid interference from the evaporation of iodine into the gas phase. ${ }^{16,20}$ For these initial concentrations, the product solution is a mixture of iodine and iodide, which has a lower surface tension and density than the reactant solution.

Reagent-grade chemicals (Sigma-Aldrich, Spektrum-3D, Reanal) were used throughout the work. Potassium iodate was dissolved in deionized water to obtain a $25.8 \mathrm{mM}$ solution. Arsenous acid solution of $72.2 \mathrm{mM}$ was prepared by dissolving sodium arsenite and adding sulfuric acid dropwise to set the $\mathrm{pH}$ of the final solution to 7 .

Two different reaction chambers made of quartz were used in the experiments. In one, termed open cell, the liquid was only filled up to an inner rim creating a horizontal flat liquid-air interface as shown in Fig. 1(a) and (b). In the other, termed closed cell, the liquid entirely filled the space creating a square block (see Fig. 1(c)). The reactants were premixed in 1:1 ratio as they were injected into the cells from below through four equally spaced bores by means of a double syringe pump

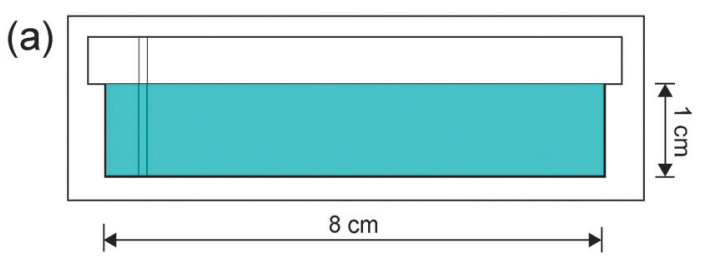

(b)

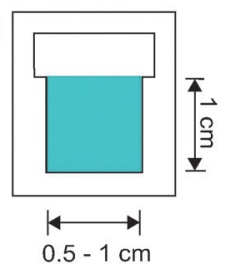

(c)

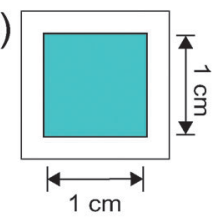

Fig. 1 Reaction chambers used in the parabolic flight. Open cell side view in the $x z$-plane (a), end view in the $y z$-plane (b). In this cell the liquid layer is in contact with an air layer (5 mm high). In panel (c) we show an end view of the closed cell. 


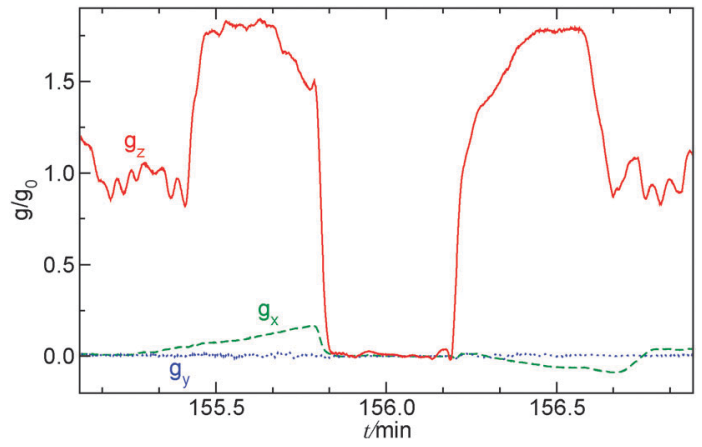

Fig. 2 Gravitational acceleration during a single parabola. The red solid curve shows the relative vertical acceleration $g_{z} / g_{0}$ during the parabolas, while the green dashed and blue dotted traces describe the acceleration along and transversal to the aircraft main axes during the parabolas.

(Harvard apparatus) so the concentration of potassium iodate was $12.9 \mathrm{mM}$ and that of arsenous acid was $36.1 \mathrm{mM}$.

During the parabolic flight campaign there were six parabola sequences, each containing five parabolas. Within a single parabola two hyper-g phases sandwiched the micro-gravity phase, where the gravitational acceleration was $1 \%$ of its normal value on ground, as shown in Fig. 2. For open cells the filling was generally performed during the 0 -g phase of the first parabola within each sequence, while for closed cells it was carried out before the parabolas. The reaction was initiated in the subsequent $0-\mathrm{g}$ phase by a short electrolysis at a pair of horizontally or vertically oriented platinum wires with a 2.5$7.0 \mathrm{~V}$ electrical potential difference between them. The reaction fronts were followed thanks to the color change in the solution when the species $\mathrm{I}_{3}{ }^{-}$forms and were monitored with a CCD camera attached to a computer. The stored images were later analyzed with in-house software, where the velocity of propagation was determined for the tip of the reaction during the various phases of gravity. For closed cells the modulation of the front shape upon the variation of gravity was observed, since the upper solid-liquid interface was unaffected by the transverse $x$ - and $y$-components of the gravitational acceleration present in each parabola (see Fig. 2).

\section{Theoretical model and parameters}

To model the propagation of the chemical front in the presence of convection, we consider a two-dimensional slab of length $L_{x}$ and height $L_{z}$ in a $(x, z)$ reference frame. As sketched in Fig. 3, this represents a vertical cut in a 3-dimensional rectangular reactor, where the $z$ axis is oriented against the gravitational acceleration $\mathbf{g}=(0,-g)$ and the chemical front propagates horizontally in the $x$ direction.

In a previous paper, ${ }^{38}$ we discussed that a reduction of the 3-dimensional problem to its 2-dimensional section can be applied without any loss of information. We consider the system as isothermal since the reaction is only slightly exothermic ${ }^{21}$ and heat dissipates much faster than mass, and can thus be neglected. ${ }^{31,56}$ In our simulations, as in the parabolic flight experiments, we

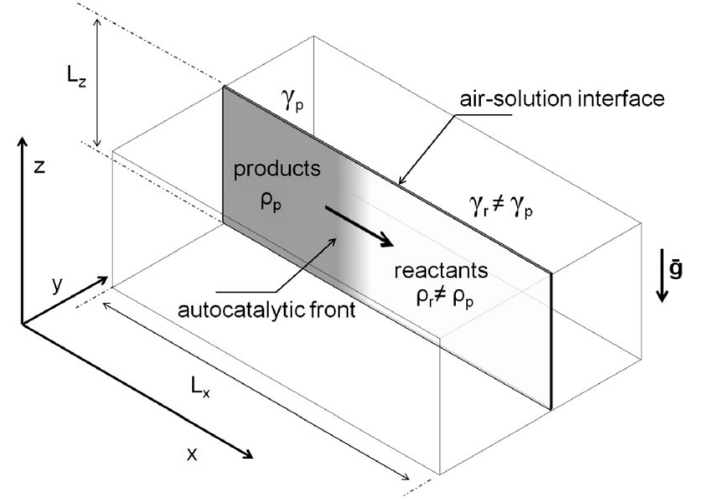

Fig. 3 Sketch of the vertical slab defining the spatial domain of our numerical description.

consider both the case of a closed reactor and the case where the vertical slab is open to the air on the top boundary (see analogous experimental setup in ref. 18). The solution of the autocatalytic product, characterized by a density $\rho_{\mathrm{p}}$ and a surface tension $\gamma_{\mathrm{p}}$, propagates towards positive $x$ (from left to right here) invading the fresh reactant solution with density $\rho_{\mathrm{r}}$ and surface tension $\gamma_{\mathrm{r}}$.

The dynamics of the traveling front obey a set of partial differential equations obtained by coupling the incompressible Navier-Stokes equations for the flow velocity $\mathbf{v}$ to a ReactionDiffusion-Convection (RDC) equation for $c$, the concentration of the autocatalytic species:

$$
\begin{gathered}
\partial_{t} c+(\mathbf{v} \cdot \nabla) c=D \nabla^{2} c+f(c), \\
\partial_{t} \mathbf{v}+(\mathbf{v} \cdot \nabla) \mathbf{v}=-\frac{1}{\rho_{0}} \nabla p+v \nabla^{2} \mathbf{v}-g \frac{\left(\rho-\rho_{0}\right)}{\rho_{0}} 1_{z}, \\
\nabla \cdot \mathbf{v}=0,
\end{gathered}
$$

where $\mathbf{v}=(u, v)^{T}$ is the velocity field and $p$ the dynamic pressure including the hydrostatic term. The kinematic viscosity $\nu$ and the diffusion coefficient of the autocatalytic species $D$ are assumed constant. These equations are derived in the Boussinesq approximation, ${ }^{57}$ assuming that density changes only affect the gravitational term of eqn (7), where $\left(\rho-\rho_{0}\right) / \rho_{0}$ is the density variation relative to the initial density of the reactant solution $\rho_{0}$. The kinetic function $f(c)=k c^{2}\left(a_{0}-c\right)$ is a cubic autocatalytic scheme capable of describing the IAA reaction in our initial reactant concentrations. ${ }^{1,5} k$ is the rate constant of the reaction and $a_{0}$ is the initial concentration of $\mathrm{IO}_{3}{ }^{-}$. Here the autocatalytic species corresponds to $\mathrm{I}^{-}$, which, even in small traces, can trigger a front propagation.

We apply no-flux boundary conditions for the concentration at the four boundaries and no-slip conditions for the velocity field at solid boundaries. To include the shear force at the free surface caused by surface tension gradients when the reactor is open, we use a Marangoni boundary condition for the horizontal component of the fluid velocity:

$$
\mu \frac{\partial u}{\partial z}=\frac{\partial \gamma}{\partial x} \quad \text { at } \quad z=L_{z}
$$

where $\gamma$ is the surface tension of the solution and $\mu$ the dynamic viscosity. We assume a linear dependence between the surface 
tension and the surfactant concentration $\left(\gamma=\gamma_{0}+(\mathrm{d} \gamma / \mathrm{d} c) c\right.$, with $\gamma_{0}$ the initial surface tension of the solution and $\mathrm{d} \gamma / \mathrm{d} c$ constant). The vertical component of the velocity $v$ is set to zero at the top border because we assume a non-deformable interface.

The equation system can be written in a dimensionless form by using the time scale of the chemical process $t_{0}=1 /\left(\mathrm{ka}_{0}{ }^{2}\right)$ and the reaction-diffusion characteristic length $L_{0}=\sqrt{D t_{0}}$. Typically, for the IAA reaction, ${ }^{1,5}$ the kinetic rate constant $k=2 \times$ $10^{4} \mathrm{M}^{-2} \mathrm{~s}^{-1}$ and $D \sim 2 \times 10^{-5} \mathrm{~cm}^{2} \mathrm{~s}^{-1} \cdot{ }^{2,21}$ Here the initial concentration of iodate is $a_{0}=12.9 \mathrm{mM}$, leading to $t_{0} \sim 0.3 \mathrm{~s}$ and $L_{0} \sim 0.002 \mathrm{~cm}$. Velocity, pressure and concentration scales are $v_{0}=L_{0} / t_{0}=\sqrt{D / t_{0}}, p_{0}=\frac{\rho_{0} L_{0} v_{0}}{t_{0}}$ and $a_{0}$, respectively. By introducing the vorticity as $\omega=\nabla \times \mathbf{v}$ and the streamfunction, $\psi$, through the relations $u=\partial_{z} \psi$ and $v=-\partial_{x} \psi$, the dimensionless form of our equations reads

$$
\begin{gathered}
\frac{\partial c}{\partial t}+\left(\frac{\partial \psi}{\partial z} \frac{\partial c}{\partial x}-\frac{\partial \psi}{\partial x} \frac{\partial c}{\partial z}\right)=\nabla^{2} c+c^{2}(1-c) \\
\frac{\partial \omega}{\partial t}+\left(\frac{\partial \psi \partial \omega}{\partial z \partial x}-\frac{\partial \psi \partial \omega}{\partial x \partial z}\right)=S_{c}\left(\nabla^{2} \omega-R a \frac{\partial c}{\partial x}\right) \\
\frac{\partial^{2} \psi}{\partial x^{2}}+\frac{\partial^{2} \psi}{\partial z^{2}}=-\omega
\end{gathered}
$$

We set the Schmidt number, $S_{c}=\nu / D=445$ since we refer to dynamics in aqueous solutions with the water kinematic viscosity $\nu=0.0089 \mathrm{~cm}^{2} \mathrm{~s}^{-1}$. The solutal Rayleigh number $R a$ is defined as

$$
R a=-\frac{1}{\rho_{0}} \frac{\partial \rho}{\partial c} \frac{a_{0} L_{0}^{3} g}{D \nu}
$$

where $\frac{1}{\rho_{0}} \frac{\partial \rho}{\partial c}$ is the solutal expansion coefficient of the density that we assume to depend linearly on the concentration of the autocatalytic product. $\frac{\partial \rho}{\partial c}<0(R a>0)$ if products are less dense than the non-reacted medium, such as in the IAA reaction. According to the experimental data reported in the literature, ${ }^{21}$ we set $R a=0.2$.

In $(\omega-\psi)$ form, the Marangoni boundary condition reads

$$
\omega=-M a \frac{\partial c}{\partial x} \quad \text { at } \quad z=L_{z},
$$

where the solutal Marangoni number $M a$ is defined as:

$$
M a=-\frac{1}{\mu \sqrt{k D}} \frac{\mathrm{d} \gamma}{\mathrm{d} c} .
$$

When, like in our case, $\frac{\mathrm{d} \gamma}{\mathrm{d} c}<0(M a>0)$, the product solution has a lower surface tension than the reactants and the front experiences a surface force along the propagation direction. According to this definition, the order of magnitude of $\mathrm{Ma}$ for our system should be $10^{4}$. Although, due to numerical instabilities, we cannot achieve this order of magnitude, in Section 4.3 we show how a numerical study with lower values of this parameter (we use $M a=10)$ can be instructive to infer reliable characteristics of the experimental system and to extrapolate dynamical properties of the front in the domain of high $M a$.

The system of eqn (10)-(12) is solved by using the Alternating Direction Implicit Method (ADI) proposed by Peaceman and Rachford. ${ }^{58}$ We consider a spatial domain of dimensionless length $L_{x}=500$ and height $L_{z}=100$ discretized over a grid of $1000 \times 400$ points. RDC-equations are integrated by using the space step $h x=0.5$. A logistic-type function $c(x, z, t)=1$ / $\left(1+\exp \left(x-x_{0}\right)\right)$, with the front tip located at $x_{0}=60$ space units along the horizontal dimension $x$, is set as the starting spatial distribution of the autocatalytic species $c(x, z, t)$ to initiate the front propagation. This is the general solution to the pure reaction-diffusion problem associated with a cubic autocatalysis, characterized by a constant shape and a front width $w_{\mathrm{RD}}=13 . .^{1,5}$ Calculations are run for 55 time units $(110 \mathrm{~s})$, using the integration time step $h t=1 \times 10^{-3}$. In order to simulate the time-dependent gravity changes during the parabolic flight, the gravitational term embedded in $R a$ (see eqn (13)) is modulated in analogy to the profile $g_{z} / g_{0}$ shown in Fig. 2. This step function features the normalized magnitude of the gravitational acceleration $g / g_{0}$ (where $g_{0}=9.81 \mathrm{~m} \mathrm{~s}^{-2}$ ) and approximates one parabola of the real parabolic flight traced in gray: two 22-seconds-phases of hypergravity (where $g / g_{0}=2$ ) separated by a 22-seconds low-gravity phase (with $g / g_{0} \rightarrow 0$ ).

\section{Results and discussion}

\subsection{Experimental results}

In the iodate-arsenous acid reaction the product solution is less dense than fresh reactants, i.e. the net density change is negative. Therefore in the presence of gravity for a reaction front propagating horizontally in a closed cell, the product solution tends to advance on the top of the denser reactant solution giving rise to a single convection roll. With a forward fluid flow on the top and a reverse fluid flow on the bottom, the originally vertical reaction front becomes tilted as shown in Fig. 4. As a result of the local mixing, the front takes on a velocity of propagation greater than that for a pure reaction-diffusion system.

The effect of the variation in the gravitational acceleration is visible in the deformation of the tip of the reaction front. The

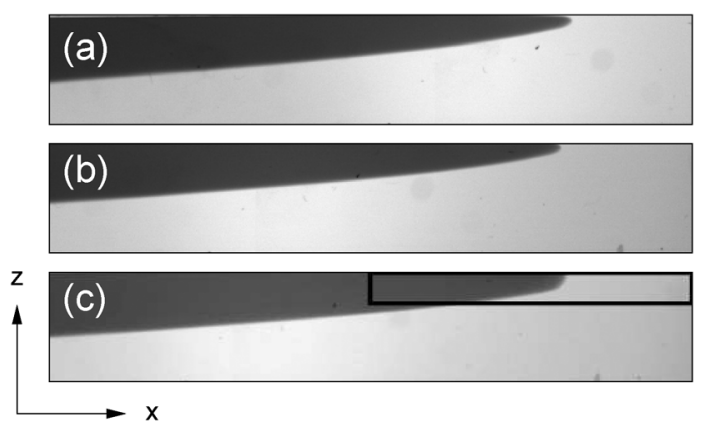

Fig. 4 Images of the reaction front in a closed reactor at $g_{z} / g_{0}=1.0$ (a), 1.7 (b) and 0.01 (c). The black rectangle in (c) is the window where the front evolution of the tip was monitored to construct Fig. 5. Field of view: $5.8 \mathrm{~cm} \times 1.0 \mathrm{~cm}$. 


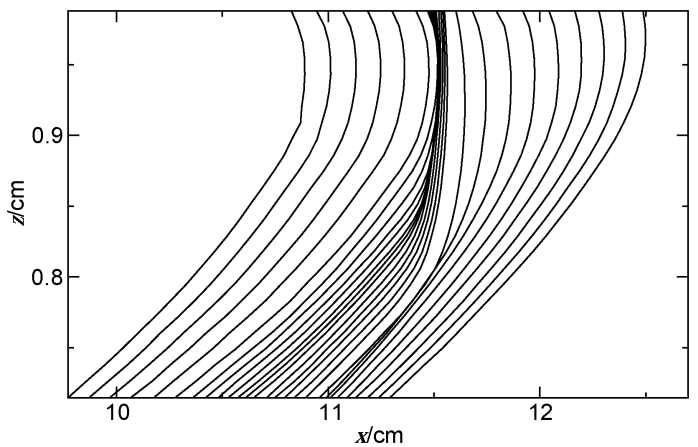

Fig. 5 Spatio-temporal evolution of front profiles with $\Delta t=2 \mathrm{~s}$ for a reaction front propagating from left to right in a closed cell within the black window shown in Fig. 4(c) as the plane enters the micro-g phase from the hyper-g phase and back again.

front positions determined for a sequence of images are shown in Fig. 5 for a closed cell during a single parabola. The advance in the upper layer abruptly stops as the airplane enters the micro-g phase, where convection ceases to exist. Diffusion remains the sole transport process leading to the decrease in the velocity of propagation. For a thin reaction-diffusion front the velocity of propagation $v$ depends on the curvature ${ }^{2}$ according to

$$
v=v_{0}+D \kappa
$$

where $v_{0}$ is the velocity of the planar front and $\kappa$ is the curvature with sign convention in accordance with the direction of propagation. The slowing down is therefore more significant in the advanced upper layer, where the front segment is characterized by a smaller radius of curvature. As soon as the plane enters the hyper-g phase again, the fluid starts to flow forward on the top, tilting the front profile further. As the position of the tip of the reaction front presented in Fig. 6 shows, in each phase of the flight the reaction front propagates at a constant velocity following a short transition period.

For an open cell similar convective patterns arise in the normal-g and hyper-g phases of the flight, the less dense reactant solution tends to advance on the top tilting the reaction front. Entering the micro-g phase however, the convective motion does not stop entirely. The propagation at the top is faster than that for

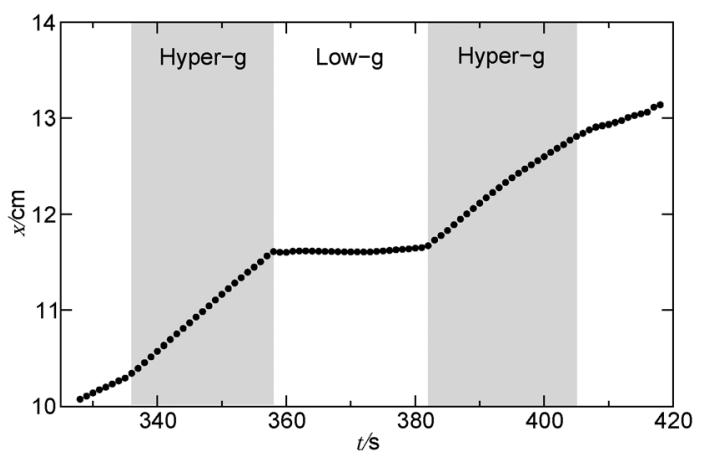

Fig. 6 Position of the tip of the reaction front in a single parabola in a closed reactor. The relative gravity is shown in the acceleration profile in Fig. 2.

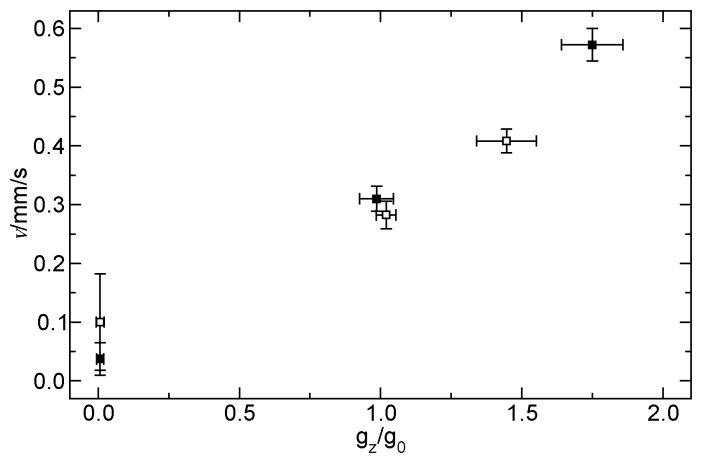

Fig. 7 Velocity of the front propagation as a function of the acceleration of gravity for closed cells (filled squares) and for open cells (open squares).

the pure reaction-diffusion fronts of the closed cell. At the reaction front a concentration gradient of the surface active iodine builds up which leads to a surface force resulting in a forward fluid flow invariant to the changes in the gravitational acceleration. It acts in the same direction as the tilting due to the gravity, therefore its effect is only apparent in the micro-g phase as shown by the velocity of propagation as a function of $g$ in Fig. 7 .

\subsection{Numerical simulations}

The typical numerical spatio-temporal patterns driven by the RDC-coupling under modulated gravitational field are shown in Fig. 8-10. Here we illustrate snapshots of the concentration map of the autocatalytic species during successive phases of the parabolic flight: pure buoyancy (Fig. 8, analogous of experiments in closed cells), pure Marangoni (Fig. 9, analogous of experiments carried out in the absence of gravity in open cells) and combined buoyancy and Marangoni (Fig. 10, which are comparable with experiments performed in open cells on earth)

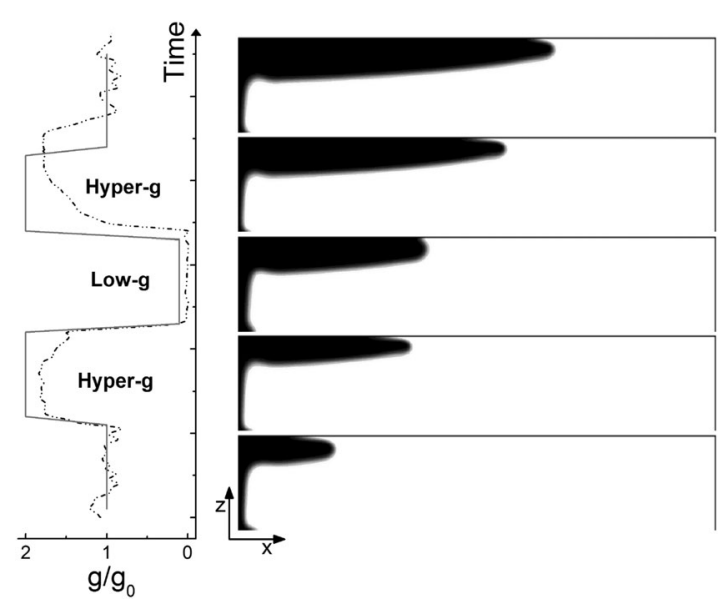

Fig. 8 Simulated spatio-temporal evolution of the autocatalytic front in a closed reactor during one parabola of the parabolic flight. The convective flow is solely driven by buoyancy effects $(R a=0.2, M a=0)$. The snapshots show the concentration map of the autocatalytic species $c$ at $t=22 \mathrm{~s}, 44 \mathrm{~s}$, $66 \mathrm{~s}, 88 \mathrm{~s}$ and $110 \mathrm{~s}$ (from bottom to top), during the successive phases of the parabolic profile illustrated in the left panel. The front is observed to slow down in micro-gravity and accelerate in hyper-g. A reverse flow is observed close to the air-solution interface. 


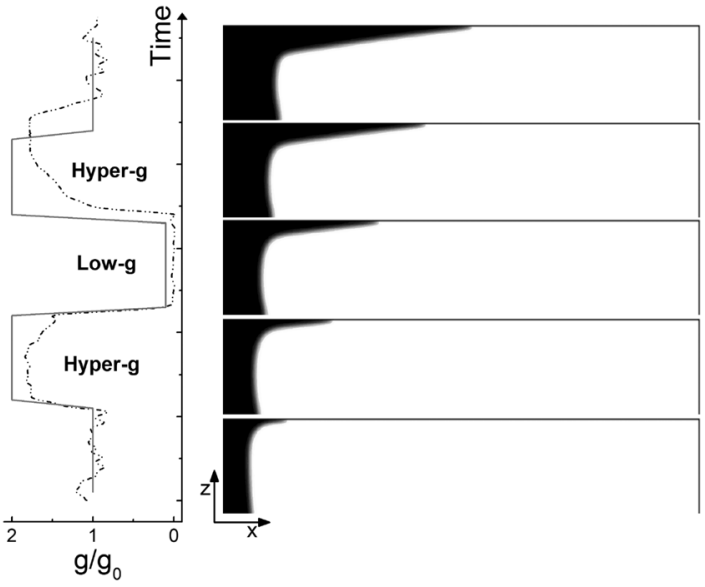

Fig. 9 Same as in Fig. 8 but when only Marangoni effects are operative $(R a=0$, $M a=10$ ). The front propagates at a constant speed and is unaffected by changes in the amplitude of gravity. No return flow is observed at the surface.

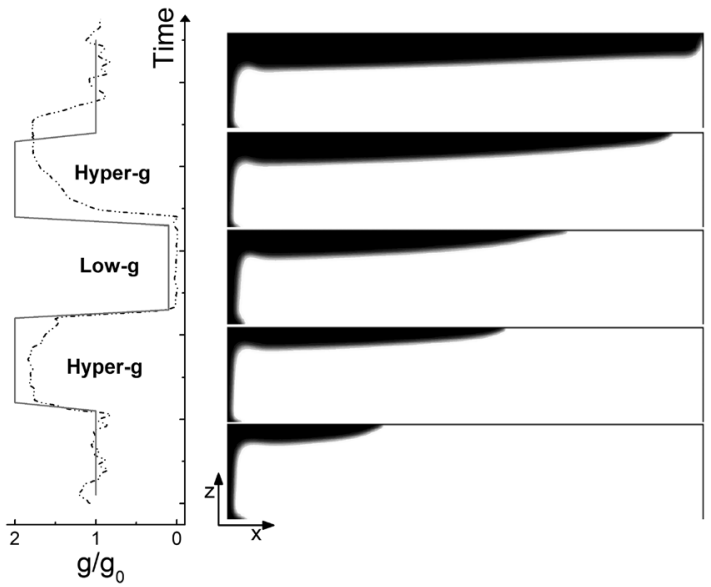

Fig. 10 Same as in Fig. 8 but when the hydrodynamic flows are driven by the cooperative contribution of buoyancy and Marangoni effects $(R a=0.2$, $M a=10$ ). The dynamics are a combination of those of Fig. 8 and 9, i.e. a slowing down of the buoyancy effect is obtained in the micro-gravity phase but no return flow is obtained as in the Marangoni-driven flow field.

contributions to the hydrodynamic motions which modify the RD-travelling interface between the reacted solution on the left (in black) and the non-reacted substrate on the right (in white).

We also quantitatively characterized the response of the front dynamics to the modulated gravity by following the front mixing length $L_{\mathrm{m}}$ as a function of time (Fig. 11(b)),

$$
L_{\mathrm{m}}=x_{\text {tip }}-x_{\text {back }}
$$

which measures the distance between the tip and the back of the traveling front. The definition of $x_{\text {tip }}$ and $x_{\text {back }}$ is based on the transversely averaged value of the concentration profile $c(x$, $z, t$ ) along the $z$-direction:

$$
\langle c\rangle(x, t)=\frac{1}{L_{z}} \int_{0}^{L_{z}} c(x, z, t) \mathrm{d} z .
$$

The tip localizes the position along $x$ in front of which the depth-averaged concentration is less than 0.01 , while the back
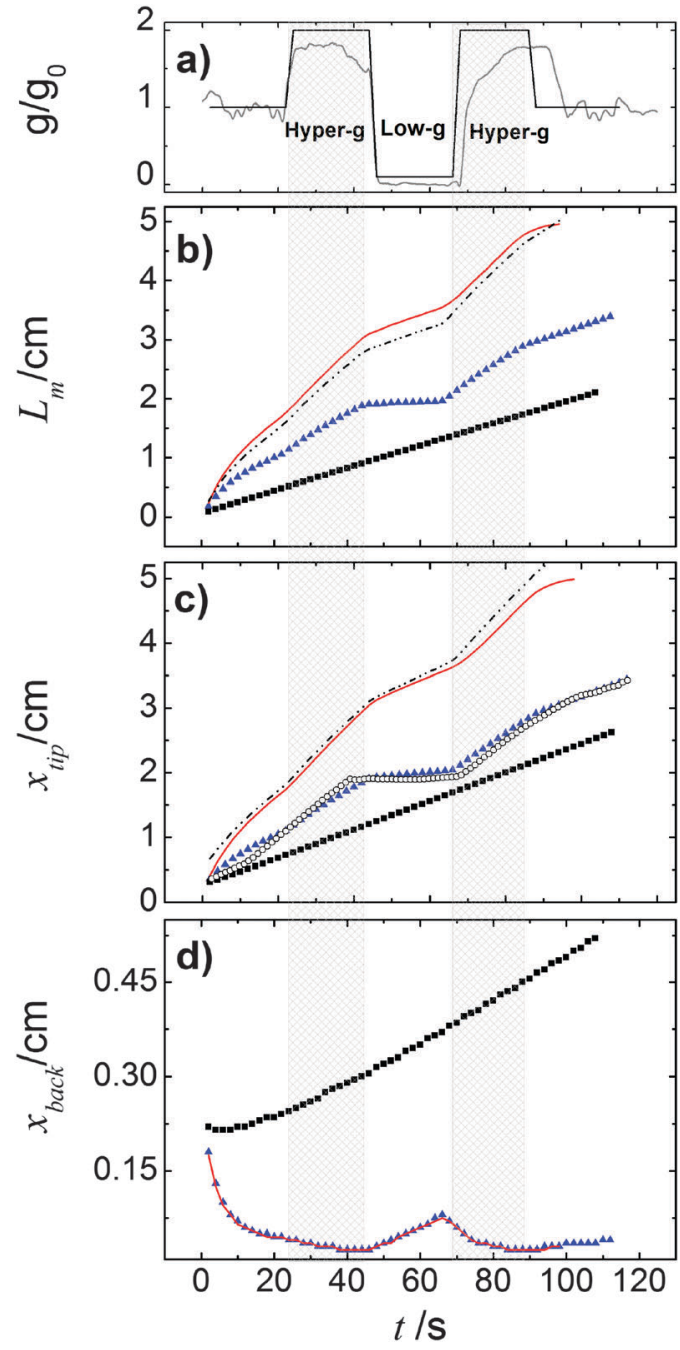

Fig. 11 Characterization of the front response to the modulated gravitational term $\mathrm{g} / \mathrm{g}_{0}$. In (a) we report in gray the parabolic profile followed during the real flight while its step function approximation used in our simulations is shown in black; in (b) we plot the mixing length $L_{m}$ as a function of the time; in (c) and (d) the two contributions to mixing length, $x_{\text {tip }}$ and $x_{\text {back }}$ are reported versus time. In each panel blue triangles, black squares and red solid curves follow the system dynamics for the pure buoyancy-driven, the pure Marangoni-driven and buoyancy-Marangonidriven convection, respectively. The black dashed curves in panels (b) and (c) trace a linear combination of the pure buoyancy and pure Marangoni characteristics. Empty circles in panel (c) represent the experimental trend of Fig. 6 suitably shifted to the same position range as in the simulations.

corresponds to the position behind which the depth-averaged concentration is larger than 0.99 . To better understand changes in the mixing length evolution, $x_{\text {tip }}$ and $x_{\text {back }}$ as a function of time are also reported in Fig. 11(c) and (d).

When the reaction is carried out in a closed reactor, the hydrodynamic flow is induced by the propagation of the less dense autocatalytic species over the denser fresh reactants, which determines a clockwise vortex localized around the reactive interface and co-moving with it. ${ }^{35}$ The resulting RDC-structure is elongated towards the top and the intensity of this deformation follows the magnitude of the gravitational term $g / g_{0}$ as depicted in Fig. 8 . 
In the first part of the dynamics where $g / g_{0}$ equals 1 , the chemical front starts tilting in the upper layer of the reactor and the lower part of the interface moves back until the side wall of the reactor is reached. A short induction period in which $x_{\text {tip }}(t)$ slowly grows and $x_{\text {back }}(t)$ decays to zero is described by the blue triangles of Fig. 11(c) and (d). Accordingly, during this initial phase the front mixing length exhibits a monotonically increasing transient scaling with the square root of time (see the initial trend traced by blue triangles in Fig. 11(b)), as characterized in previous studies. ${ }^{35,49}$ However, differently from what was found in these earlier studies, here the profile of the autocatalytic front cannot stabilize over a constant shape since the boundary conditions at the left lateral border hinder the formation of the asymptotically stable symmetrical shape.

The increasing buoyancy forcing in the first hyper-g phase further sustains the front deformation at the top of the reactor with a parallel increment of the front speed. The time interval of the hyper-g step is large enough to let the front speed stabilize over a new regime as demonstrated by the almost constant slope of $L_{\mathrm{m}}(t)$ and $x_{\text {tip }}(t)$ within this region.

The dynamics during the low-g phase tend to recover the peculiarities of a $\mathrm{RD}$-structure ${ }^{2}$ and this is reflected in the increased extent of the reacted region around the front tip where no preferential growth direction dominates. Also note that within this interval, the back front experiences a temporary increment along the horizontal axis (see Fig. 11). In agreement with experimental data, this flight phase is characterized by a sharp drop of the front speed as shown by the reduced slope of the mixing length and the front tip curves in Fig. 11(b) and (c).

During the transition to the new hyper-g phase, the autocatalytic front is again pushed forward along the top part of the slab and backward at the bottom. In parallel, we can appreciate an increase in the mixing length with the slope of the curve $L_{\mathrm{m}}(t)$ and $x_{\text {tip }}(t)$ that match the same values as in the first hyper$\mathrm{g}$ episode in the parabola.

We compared the trends observed in the closed reactor with those obtained with the analogous system where only surface effects are at play (see Fig. 9). When the reaction is performed in an open vessel, one of the main products of the autocatalytic process, $\mathrm{I}_{2}$, lowers the surface tension at the interface between the reacted and the non-reacted solution so promoting the conditions for Marangoni-driven convection. The net force at the air-liquid interface enhances the preferential growth of the front at the contact line between solution and air towards the right side of the slab and determines a morphological change in the front shape. While in the presence of pure buoyancydriven convective motions the front profile along the $z$-axis shows a non monotonic contour with an evident maximum at the slab top, in the same region, when only surface effects rule the spatio-temporal dynamics of the front, a monotonouslyshaped interface separates the reacted solution from fresh reactants. This is clear when comparing the spatio-temporal patterns in Fig. 8 with those in Fig. 9 and is due to the different topology of the flow field related to the two separate sources of convection. These aspects are extensively discussed in ref. 37, 45 and 49. As expected, the system controlled by pure Marangoni-driven convection is not affected by the modulated gravitational field and follows the same qualitative behavior illustrated in ref. 45 where no dynamical changes in the gravitational term are considered. All the topological observables of the front (see black squares in Fig. 11(b) and (c)) show a monotonic increase in time. The front moves with a constant speed which can be extrapolated from the slope of the linear trend described by the black squares of Fig. 11(c).

On the other hand, a similar dependence of the chemical field on the gravitational parabolas as in the pure buoyancy case is preserved and becomes even more evident when the onset of hydrodynamic motions are promoted by the buoyancy-Marangoni coupling (see Fig. 10). Here, the emergent convective instability is the result of a cooperative interplay between buoyancy- and Marangoni-induced flows, which are both oriented along the front propagation direction. In turn, the front shape merges the properties of the pure buoyancy and the pure Marangoni cases (compare Fig. 10 with Fig. 8 and 9): the front profile is dominantly pulled to the right of the cell at the air-solution interface but also experiences a deformation against the gravitational field. The extent of this deformation depends upon the intensity of the term $g_{z} / g_{0}$ and the response of $L_{\mathrm{m}}(t), x_{\text {tip }}(t)$ and $x_{\text {back }}(t)$ to its variations is quantitatively followed by the red curves in Fig. 11(b)-(d), respectively. Both $L_{\mathrm{m}}(t)$ and $x_{\text {tip }}(t)$ are shifted to higher values with respect to the limiting pure buoyancy- and pure Marangoni-controlled systems. It is worth noticing that the direct sum of $x_{\text {tip }}(t)$ of the pure buoyancy and pure Marangoni cases (see dashed curve in Fig. 11(c)) closely follows the trend of the coupled Marangoni-buoyancy system drawn in red in the same graph. This feature is instructive to get reliable insights into the front properties for large values of $M a$, prohibitive for a direct simulation. As described in a previous study, ${ }^{46}$ when the sole Marangoni contribution is controlling the onset of convection in the system, $x_{\text {tip }}$ preserves a linear temporal evolution even for large $M a\left(\sim 10^{3}\right)$, with a propagation speed (the slope of the line $\left.x_{\text {tip }}(t)\right)$ scaling with $M a$ as $v=0.43 \sqrt{M a}$ in dimensionless units (corresponding to $3.5 \times 10^{-3} \sqrt{\mathrm{Ma}} \mathrm{cm} \mathrm{s}^{-1}$ ). Since buoyancy and Marangoni contributions are likely to be still additive in the domain of high $M a$, we can extrapolate $x_{\text {tip }}$ dynamics for $M a \sim 10^{4}$ as a linear combination of the blue trends in Fig. 11(b) and (c), and a line with slope $0.35 \mathrm{~cm} \mathrm{~s}^{-1}$. This is also true for $L_{\mathrm{m}}$ trajectories, since these are mainly determined by the $x_{\text {tip }}$ dynamics. By contrast, the $x_{\text {back }}$ dynamics are pretty much the same when both contributions are at play and for the pure buoyancy case (compare blue and red curves in Fig. 11(d)).

\subsection{Discussion}

A comparison between Fig. 4 and 8 as well as Fig. 6 and 11 (with the data of Fig. 6 inserted as empty circles in Fig. 11(c) after a suitable shift to the same position range as in the simulations) shows that modulated buoyancy effects are clearly operational in the experiments. Indeed the front deformation is reduced and the front velocity slows down when the system enters the micro-gravity phase. As simulations (see Fig. 9) suggest that the density and surface-tension effects can be considered in a good approximation to be additive in the deformation of the front, 
the measurement of the difference in velocity at $g_{z} / g_{0} \sim 0$ is a way to extrapolate the magnitude of the Marangoni effects.

The deformation of the front tip observed in the experiments for closed cells has been successfully reproduced in the calculations. Note how both in the experiments (Fig. 4) and numerical simulations (Fig. 8) the convex region around the front tip shrinks when the system enters the hyper-gravity and swells in the low-gravity phases, where the dynamics recall the features of a pure autocatalytic RD-front. The variation in the velocity of the tip propagation during the various phases also reveals the good agreement between experiments and calculations. In order to achieve a similar comparison for open cells, the analysis requires a longer time span of micro-gravity uninterrupted by hyper-gravity phases, as is available in a suborbital sounding rocket flight.

\section{Conclusions}

By measuring the periodic changes from an accelerated front propagation under hyper-gravity towards a slowed down propagation under low-gravity we have shown that gravity markedly influences the motion of autocatalytic reaction fronts even in thin fluid layers. This behavior is caused by the amplification or decay of a buoyancy-driven vortex surrounding the front when increasing or decreasing the g-level.

Numerical simulations of the problem are in good quantitative agreement with experimental measurements of closed cells made on board of a parabolic flight in which the gravity field is modulated periodically. They further show that density and surface tension driven effects are in a good approximation additive so our method can be used to infer dynamics at larger Marangoni numbers and it is appropriate for future predictions in open cells. This is also useful to extrapolate more quantitative information on the magnitude of each effect alone in the evolution of the front on earth.

\section{Acknowledgements}

We thank Ulrike Strachauer, Eszter Orosz, and Armin Heinze for their support before and during the parabolic flight campaign. Financial support by ESA, WBI, TÉT, ESTEC, Prodex, DLR (50WM1144), Regione Sardegna (POR 2007-2013) and DFG is gratefully acknowledged.

\section{References}

1 Oscillations and Traveling Waves in Chemical Systems, ed. R. J. Field and M. Burger, Wiley, New York, 1985.

2 I. R. Epstein and J. A. Pojman, An introduction to nonlinear chemical dynamics, Oxford University Press, Oxford, 1998.

3 W. van Saarloos, Phys. Rep., 2003, 386, 29.

4 T. A. Gribschaw, K. Showalter, D. L. Banville and I. R. Epstein, J. Phys. Chem., 1981, 85, 2152.

5 A. Hanna, A. Saul and K. Showalter, J. Am. Chem. Soc., 1982, 104, 3838.
6 G. Bazsa and I. R. Epstein, J. Phys. Chem., 1985, 89, 3050.

7 I. Nagypál, G. Bazsa and I. R. Epstein, J. Am. Chem. Soc., 1986, 108, 3635.

8 J. A. Pojman and I. R. Epstein, J. Phys. Chem., 1990, 94, 4966.

9 H. Miike, H. Yamamoto, S. Kai and S. C. Müller, Phys. Rev. E: Stat. Phys., Plasmas, Fluids, Relat. Interdiscip. Top., 1993, 48, 1627.

10 M. J. B. Hauser and R. H. Simoyi, Chem. Phys. Lett., 1994, 227, 593.

11 O. Inomoto, T. Ariyoshi, S. Inanaga and S. Kai, J. Phys. Soc. Jpn., 1995, 64, 3602.

12 K. Matthiessen, H. Wilke and S. C. Müller, Phys. Rev. E: Stat. Phys., Plasmas, Fluids, Relat. Interdiscip. Top., 1996, 53, 6056.

13 G. Schuszter, T. Tóth, D. Horváth and Á. Tóth, Phys. Rev. E, 2009, 79, 016216.

14 I. Bou Malham, N. Jarrige, J. Martin, N. Rakotomalala, L. Talon and D. Salin, J. Chem. Phys., 2010, 133, 244505.

15 T. Rica, E. Pópity-Tóth, D. Horváth and Á. Tóth, Phys. D, 2010, 239, 831.

16 E. Pópity-Tóth, D. Horváth and Á. Tóth, J. Chem. Phys., 2011, 135, 074506.

17 O. Miholics, T. Rica, E. Pópity-Tóth, D. Horváth and Á. Tóth, J. Chem. Phys., 2011, 135, 204501.

18 L. Sebestíková and M. J. B. Hauser, Phys. Rev. E, 2012, 85, 036303.

19 F. Rossi, M. A. Budroni, N. Marchettini and J. CarballidoLandeira, Chaos, 2012, 22, 037109.

20 E. Pópity-Tóth, V. Pimienta, D. Horváth and Á. Tóth, J. Chem. Phys., 2013, 139, 164707.

21 J. A. Pojman, I. R. Epstein, T. J. McManus and K. Showalter, J. Phys. Chem., 1991, 95, 1299.

22 J. Masere, D. A. Vasquez, B. F. Edwards, J. W. Wilder and K. Showalter, J. Phys. Chem., 1994, 98, 6505.

23 M. Böckmann and S. C. Müller, Phys. Rev. Lett., 2000, 85, 2506.

24 A. De Wit, Phys. Rev. Lett., 2001, 87, 054502.

25 D. Horváth, T. Bánsági Jr. and Á. Tóth, J. Chem. Phys., 2002, $117,4399$.

26 T. Bánsági Jr., D. Horváth, Á. Tóth, J. Yang, S. Kalliadasis and A. De Wit, Phys. Rev. E, 2003, 68, 055301(R).

27 M. C. Rogers and S. W. Morris, Phys. Rev. Lett., 2005, 95, 024505.

28 T. Tóth, D. Horváth and Á. Tóth, Chem. Phys. Lett., 2007, 442, 289.

29 L. Šebestíková, J. D’Hernoncourt, M. J. B. Hauser, S. C. Müller and A. De Wit, Phys. Rev. E, 2007, 75, 026309.

30 T. Plesser, H. Wilke and K. H. Winters, Chem. Phys. Lett., 1992, 200, 158.

31 D. A. Vasquez, J. M. Littley, J. W. Wilder and B. F. Edwards, Phys. Rev. E: Stat. Phys., Plasmas, Fluids, Relat. Interdiscip. Top., 1994, 50, 280.

32 H. Wilke, Phys. D, 1995, 86, 508.

33 Y. Wu, D. A. Vasquez, B. F. Edwards and J. W. Wilder, Phys. Rev. E: Stat. Phys., Plasmas, Fluids, Relat. Interdiscip. Top., 1995, 51, 1119.

34 V. Pérez-Villar, A. P. Muñuzuri and V. Pérez-Muñuzuri, Phys. Rev. E: Stat. Phys., Plasmas, Fluids, Relat. Interdiscip. Top., 2000, 61, 3771. 
35 L. Rongy, N. Goyal, E. Meiburg and A. De Wit, J. Chem. Phys., 2007, 127, 114710.

36 M. A. Budroni, M. Masia, M. Rustici, N. Marchettini and V. Volpert, J. Chem. Phys., 2009, 130, 024902.

37 L. Rongy and A. De Wit, J. Chem. Phys., 2009, 131, 184701.

38 L. Rongy, G. Schuszter, Z. Sinkó, T. Tóth, D. Horváth, Á. Tóth and A. De Wit, Chaos, 2009, 19, 023110.

39 N. Jarrige, I. Bou Malham, J. Martin, N. Rakotomalala, D. Salin and L. Talon, Phys. Rev. E, 2010, 81, 066311.

40 Z. Dagan and L. M. Pismen, J. Colloid Interface Sci., 1984, 99, 215.

41 L. M. Pismen, J. Colloid Interface Sci., 1984, 102, 237.

42 Z. Dagan and C. Maldarelli, Chem. Eng. Sci., 1987, 42, 1259. 43 L. M. Pismen, Phys. Rev. Lett., 1997, 78, 382.

44 A. Pereira, P. M. J. Trevelyan, U. Thiele and S. Kalliadasis, Phys. Fluids, 2007, 19, 112102.

45 L. Rongy and A. De Wit, J. Chem. Phys., 2006, 124, 164705. 46 L. Rongy, A. De Wit and G. M. Homsy, Phys. Fluids, 2008, 20, 072103.

47 L. Rongy, P. Assemat and A. De Wit, Chaos, 2012, 22, 037106.

48 M. Diewald, K. Matthiessen, S. C. Müller and H. R. Brand, Phys. Rev. Lett., 1996, 77, 4466.
49 M. A. Budroni, L. Rongy and A. De Wit, Phys. Chem. Chem. Phys., 2012, 14, 14619.

50 J. Martin, N. Rakotomalala, L. Talon and D. Salin, Phys. Rev. $E, 2009,80,055101(\mathrm{R})$.

51 Y. Shi, K. Eckert, A. Heinze and M. Acker, 17th ESA Symposium on European Rocket and Balloon Programmes and Related Research, 30 May-2 June 2005, Sandefjord, Norway, 2005, pp. 545-550.

52 Y. Shi and K. Eckert, Chin. J. Chem. Eng., 2007, 15, 748-753.

53 K. Eckert, L. Rongy and A. De Wit, Phys. Chem. Chem. Phys., 2012, 14, 7337.

54 S. Fujieda, Y. Mogamia, A. Furuya, W. Zhang and T. Araiso, J. Phys. Chem. A, 1997, 101, 7926.

55 S. Fujieda, Y. Mori, A. Nakazawa and Y. Mogami, Adv. Space Res., 2001, 28, 537.

56 C. Almarcha, P. M. J. Trevelyan, P. Grosfils and A. De Wit, Phys. Rev. E, 2013, 88, 033009.

57 J. Boussinesq, Théorie Analytique de la Chaleur, GauthierVillars, Paris, 1903, vol. 2.

58 D. W. Peaceman and H. H. Rachford, J. Soc. Ind. Appl. Math., 1955, 3, 28. 